Journal of Case Reports 2018;8(2):141-143

\title{
Erythema Multiforme: Extremely Rare Side Effect of Pyrazinamide
}

\author{
Salauddin, Nafees Ahmad Khan, Rameez Najeeb, Rakesh Bhargva, Mohammad Shameem, Arif \\ Department of Pulmonary Medicine, J.N. Medical College, AMU Aligarh. Uttar Pradesh, India.
}

\author{
Corresponding Author: \\ Dr. Nafees Ahmad Khan \\ Email: nafees.doc@gmail.com \\ This is an Open Access article distributed \\ under the terms of the Creative Commons \\ Attribution License (creativecommons.org/ \\ licenses/by/3.0). \\ Received : January 16, 2018 \\ Accepted : April 24, 2018 \\ Published : May 30, 2018
}

\begin{abstract}
Background: There are various types of cutaneous drug reactions with anti-tubercular therapy (ATT), and probably each and every first line ATT can manifest these reactions. Erythema multiforme (EM) is one of the extremely rare, acute hypersensitivity reaction to various drugs including pyrazinamide. Case Report: We report a case of 19 years old female with pulmonary tuberculosis who developed biopsy proven EM 45 days after initiation of ATT, as an adverse effect of pyrazinamide. Conclusion: Physicians must be aware of this rare side effect of pyrazinamide.
\end{abstract}

Keywords: Drug-Related Side Effects and Adverse Reactions, Erythema Multiforme, Pyrazinamide, Tuberculosis.

\section{Introduction}

Drug eruptions can present with variety of dermatological manifestations, among them most common are pruritus, urticaria, angioedema, photosensitivity, fixed drug eruptions, erythema multiforme (EM), and life threatening Stevens Johnson syndrome [1]. EM is an acute inflammatory hypersensitivity reaction characterized by reddish edematous maculopapular and bullous lesions of the skin [2].

\section{Case Report}

A 19-year-old female from low socioeconomic status presented to us with complaints of severe cough with yellowish expectoration, fever, loss of appetite and weight since last one month. X-ray chest revealed infiltration in left upper and mid zone with underlying cavitations, and sputum testing for acid-fast bacillus (AFB) was positive. Anti-tubercular therapy (ATT) was started under directly observed treatment, short course (DOTS) category I according to her weight $38 \mathrm{~kg}$. X-ray chest [Fig.1] showed persistent lesions and daily regimens of HRZE according to weight were started with antiemetic and antacids. Patient followed up every 15 days and was improving clinically and radiologically. After 45 days of treatment, she complained of acute onset extremely pruritic maculopapular rashes over abdomen and lower limbs, mostly on extensor surfaces with target lesions with extreme burning sensations [Fig.2a,2b]. Total leukocyte count was mildly raised by 12000 cells $/ \mathrm{mm}^{3}$, differential of neutrophils 38, lymphocytes: 20, eosinophils: 40, basophils: 2; hemoglobin: $9.5 \mathrm{gm} \%$, absolute eosinophils count: 2000 cells $/ \mathrm{mm}^{3}$. Peripheral blood picture showed mild hypochromic with plenty of scattered eosinophils [Fig.3]. HIV screening was found to be negative, other conditions causing skin rashes like autoimmune disorders, environmental toxins, infections and insect bites were ruled out. Skin biopsy taken by dermatologist revealed eosinophils and neutrophilic infiltration at dermoepidermal junction with a focus of intra-epidermal abscess suggestive of erythema multiforme [Fig.4]. ATT drugs were discontinued by dermatologist and $40 \mathrm{mg} /$ day prednisolone started for 7 days. She improved clinically and rashes slowly subsided with brown scaly exfoliations [Fig.5]. ATT was restarted after one week serially with ethambutol, then isoniazid, then rifampicin and pyrazinamide. 


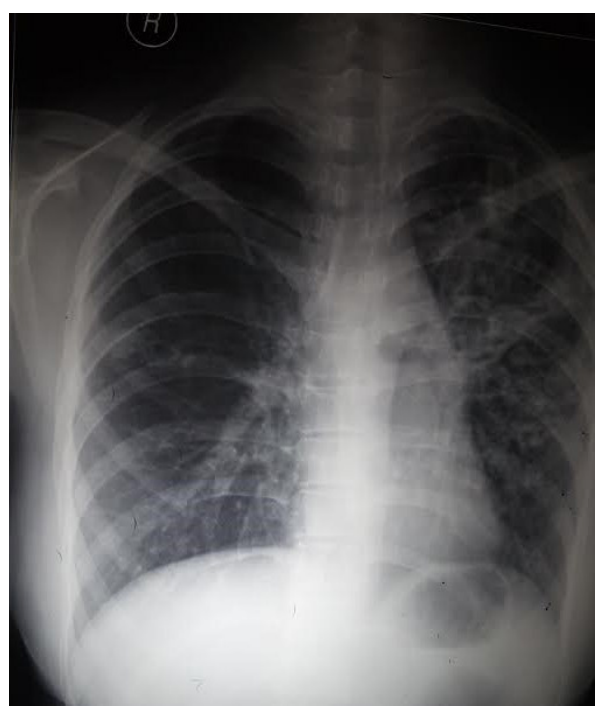

Fig.1: X-ray chest: left upper and mid zone infiltrates with cavitation.
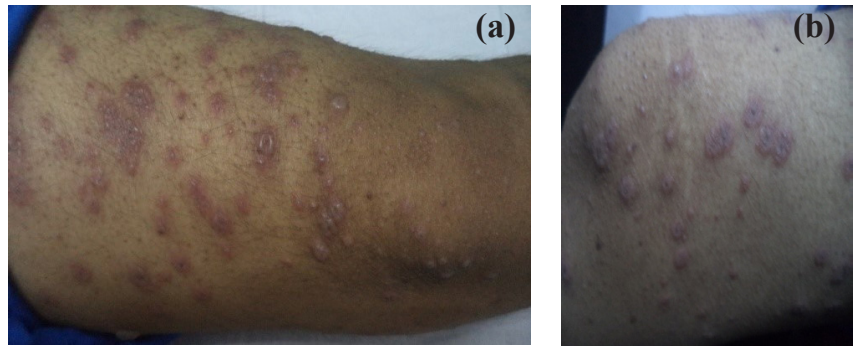

Fig.2(a): Maculopapular rashes over extensor surface of lower limb. (b): Sharply defined target lesions.

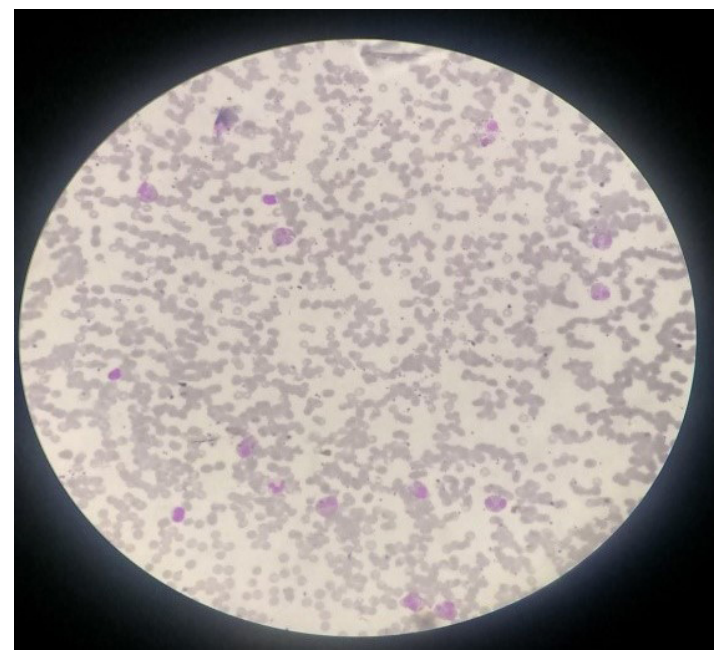

Fig.3: Blood picture showing scattered eosinophils.

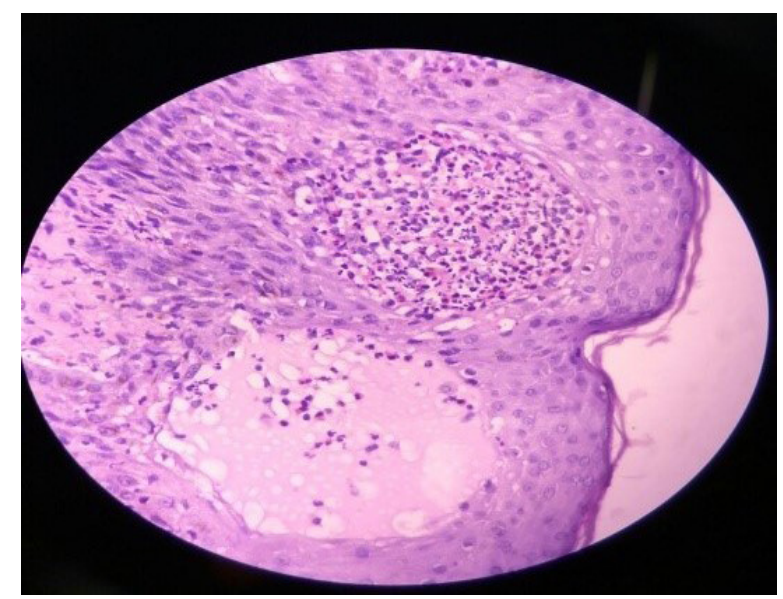

Fig.4: Skin biopsy showing eosinophilic and neutrophilic infiltrates and focus of abscess.

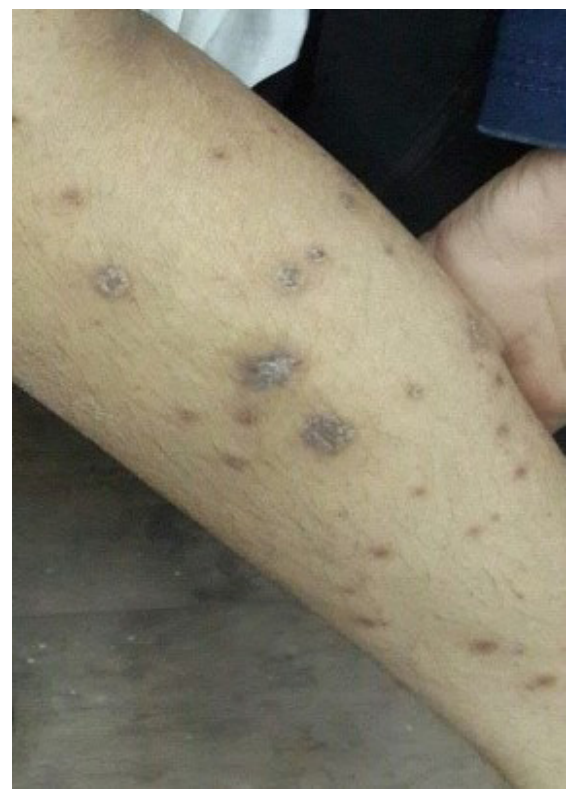

Fig.5: Healing lesion with dry scaly exfoliation.

After day one of starting pyrazinamide, patient again presented with extreme pruritus without fresh lesion, pyrazinamide was stopped again, and only HRE continued with good compliance. As patient had completed almost two month of intensive phase of ATT, only HRE was continued. The patient is improving well clinically and radiologically. 


\section{Discussion}

EM is a self limiting disease characterized by mild or no prodromal symptoms [3]. Symptomatic patients mainly presents with rapid producing numerous sharply demarcated bright red or pink macules and papules with hot burning sensation along with pruritus $[4,5]$ within 72 hours of drug intake or insult [6] with crusting or blistering sometimes occurs in the centre known as "target" or "iris" lesion [7]. EM resolves spontaneously in three to five weeks without sequelae. Diagnosis is usually clinical but skin biopsy needed to confirm diagnosis which reveals eosinophilic and lymphocytic infiltration and ballooning degeneration in the dermo-epidermal junction, dermal perivascular monocytic infiltration in the upper dermis, edema in the dermal papilla, mixed epidermal changes (vacuolar degeneration of the basal layer, satellite cell necrosis), and dermal changes (perivascular lymphocytic infiltration) [8].

Globally, total frequency of erythema multiforme is estimated at approximately 1.2-6 cases per million individuals per year, among them frequency due to only pyrazinamide is extremely rare [9]. In our case maculopapular rash appeared late in 45 days after initiation of ATT, in contrast to 72 hours as described in literature. Author fails to explain this delayed presentation of this adverse effect. All drugs were withheld and patient was kept on oral steroid prednisolone $40 \mathrm{mg} / \mathrm{kg}$ and oral anti-histamines. Patient improved clinically and rashes slowly subsided in five days with brown scaly exfoliations. ATT was started after one week serially with ethambutol, then isoniazid, then rifampicin and pyrazinamide. After one day of starting pyrazinamide appearance of similar pruritic lesion gave an indication of causative agent i.e pyrazinamide and lesion subsided after stopping pyrazinamide again. Only HRE was continued with good compliance.
The common adverse effects of pyrazinamide are nausea, vomiting, hyperuricemia, hepatotoxicity, arthralgia, and flushing. Cutaneous reactions are rare includes rashes and photosensitivity [10]. Due to these reactions patients becomes non-adherent with ATT which may be a cause of treatment failure.

\section{Conclusion}

Physicians must aware of this extremely rare side effect i.e. due to pyrazinamide for proper adherence with ATT, regardless of time of presentation of adverse effect after starting ATT, as in our case presentation can be very late.

Contributors: Sal: manuscript writing, patient management; NAK, RN: manuscript editing, patient management; RB, MS, Arif: critical inputs into the manuscript, patient management. NAK will act as guarantor. All authors approved the final version of this manuscript.

Funding: None; Competing interests: None stated.

\section{References}

1. Roujeau JC, Stern RS. Medical Progress: Severe adverse cutaneous reactions to drugs. N Engl J Med. 1994;331:1272-1285.

2. Carder RK. Hypersensitivity reactions in neonates and infants. Dermatol Ther. 2005;18:160-175.

3. Habif TP. Hypersensitivity syndromes and vasculitis. In: Clinical Dermatology: A Color Guide to Diagnosis and Therapy. $4^{\text {th }}$ ed. New York, Mosby, 2004:626-634.

4. Shin HT, Chang MW. Drug eruptions in children. Curr Probl Pediatr. 2001;31:207-234.

5. Huff JC. EM and latent herpes simplex infection. Semin Dermatol. 1992;11:207-210.

6. Carder RK. Hypersensitivity reactions in neonates and infants. Dermatol Ther. 2005;18:160-175.

7. Kennett S. EM affecting the oral cavity. Oral Surg Oral Med Oral Pathol. 1968;25:366-373.

8. Hebra F. Diseases of the skin. Fagge CH (Ed), London, New Sydenham Society, 1866, Vol. I. The New Sydenham Society: London, 1866, pp.114-118.

9. Perdu D, Lavaud F, Prévost A, Deschamps F, Cambie MP, Bongrain E, et al. Erythema multiforme due to pyrazinamide. Allergy. 1996;51:340-342.

10. Mehta DK, Martin J, Costello I, Jordan B, editors. BNF 50; London: BMJ publishing group; 2005. 